\title{
INTRODUCTION
}

\section{Goals and overview of the special issue}

\author{
Bereavement: A family affair \\ Margaret Stroebe ${ }^{\mathrm{a}, \mathrm{b} *}$, Henk Schut $^{\mathrm{a}}$ and Kathrin Boerner ${ }^{\mathrm{c}}$ \\ ${ }^{a}$ Department of Clinical Psychology, Utrecht University, 3508 TC Utrecht, The Netherlands; ${ }^{b}$ Department of Clinical Psychology and \\ Experimental Psychopathology, University of Groningen, Groningen, The Netherlands; ' ${ }^{c}$ Jewish Home Lifecare, Research Institute on \\ Aging, New York, NY 10025, USA
}

(Received 15 April 2013; final version received 17 May 2013)

The loss of a loved one is an event that affects the individual, but it is as much a family affair in the sense that bereavement affects families - as well as individuals - in complex and varied ways. Yet, while there has been enormous expansion of research over the past few decades on manifestations and phenomena associated with grief and grieving of individual bereaved persons or categories of bereaved persons, research at the family level has lagged behind. With a few valuable exceptions, the available research in the latter domain has also appeared in diverse sources, scattered across different disciplines, and is thus difficult to synthesize for the interested reader. This suggests the need for synthesis and review of research on bereavement in the family context.

There are a number of reasons why we think that a family perspective is important. In addition to the fact that death of a loved one is in itself an interpersonal event, one which affects individuals who live in a social context, the actual grieving process too incorporates important interpersonal components: People do not typically grieve alone; each member of a family is affected (in different ways) by the loss of one of its members, and these persons interact with each other in the process of coming to terms with their loss. Family and individual processes can be expected to interact in intricate ways. For example, it is understood that family dynamics (e.g. conflicted relationships to other surviving family members and to the deceased) affects the course of each person's adjustment to their loss, while at the same time, the individual reaction of a family member may have ramifications that are experienced throughout the family, affecting dynamics at this interpersonal level too. Also, there are interpersonal as well as intrapersonal consequences associated with bereavement: for example, not only are there individual outcomes relating to mental and physical health, but also social ones, such as changes in family networks and characteristics of relationships. For readers coming from a background of family systems thinking, death of a family member can be regarded as a life event, which triggers role changes and new family dynamics.

Such features as those illustrated above led us to design this Special Issue of Family Science. The main purpose is to draw together research on bereavement within the family context, providing key articles to highlight major family issues in bereavement research. In our view, it is useful for both researchers and practitioners to have such a state-ofthe-art source where those who have recently contributed to scientific understanding of interpersonal loss from diverse family perspectives can share their knowledge and express their opinions on this topic. We hope that this will give direction for future research.

The Special Issue includes 11 scientific articles, addressing diverse family-related issues that we considered to be of major interest and relevance to contemporary society, ending with an article assessing the contributions to this volume. Articles either review the literature, presenting a synthesis of advances on a specific topic, or present new research findings, or describe theoretical or research approaches (e.g. an intervention protocol; instruments for assessment at the family level). Qualitative as well as quantitative approaches are included, for these go hand-in-hand in contributing to our understanding of family bereavement dynamics and manifestations.

The Special Issue is thematically structured, beginning with two overview articles in Part 2 to set the stage, covering family grief in historical and cultural perspectives. In the first article, Jalland takes us back to nineteenth century England, an important focus, particularly given the

*Corresponding author. Email: M.S.Stroebe@uu.nl 
influence of this society across the British commonwealth then and subsequently. She illustrates the family's changing role in shaping bereavement from this historical period until the later decades of the twentieth century. Notably, Jalland identifies a shift from 'expressive to silent grief', followed by a more recent revival of expressive grieving. Such changes are linked to societal and cultural differences, medical developments and political events, including the two world wars. Jalland's account offers the possibility for readers to draw comparisons with contemporary patterns of family involvement in bereavement not only in England, but across the world.

In the second article, Rosenblatt further sensitizes us to cultural differences in the family bereavement experience, showing how the family systems theory way of thinking while extending beyond an individual focus to close relational aspects - may blind one to different cultural patterns relating to bereavement in family context, even at the level of understanding what is 'family' and what is 'grief'. Three vivid examples of family/relationship systems concepts are provided, which not only illustrate how grieving occurs in specific cultures, but also may be used to inform us about grief in other cultures, including the dominant cultures in the United States and Europe, where family systems theory arose. The theory itself can benefit from cultural attunement.

A section on mutual influence of family members and their relations follows (Part 3), with articles ranging from family characteristics and dynamics in bereavement to intergenerational issues and the experience of grief across the life cycle. Walsh and McGoldrick adopt a developmentally sensitive, family systems framework to review both the short- and long-term impact of bereavement in the family. A main argument is that assessment of family bereavement processes must consider the complex network of family relationships over the life course and across time. They, therefore, include focus on the multigenerational network of relationships, which is involved in family adaptation processes. They also explore variables that contribute to risk and resilience among family members. As Walsh and McGoldrick acknowledge, there is room for further empirical testing of many of the theoretical ideas presented in this article, and they give concrete indications as to how researchers could provide empirical verification for them.

The following two articles, by Stroebe, Schut and Finkenauer and by Carr and Boerner, examine specific, different family relations. Stroebe, Schut and Finkenauer review scientific research on the impact of the death of a child on parents, ranging from individual health consequences, risk factors and coping processes, to more social approaches such as examination of the couple's relationship and couple coping. This review extends previous coverage to include interactive coping processes among the interpersonal processes. They describe their research on regulation of emotions for the sake of the partner, a phenomenon that regularly occurs with families. Their review underscores the social as well as the personal nature of grief and illustrates how adoption of one or the other approach may actually cause different and unexpected conclusions to be reached.

By contrast, in the following article, Carr and Boerner focus on intergenerational relations in later life. Using a rich and unique prospective US data set on older couples, they examine the impact of late-life spousal bereavement on parent-child relationships. They selected another interpersonal variable, quality of the relationship with the deceased partner, to investigate links between marital quality and post-loss parent-child relationships, finding important differences compared with still-married spouses. This study is unique in showing how the impact of spousal loss on parent-child relations varies according to the nature of the late marriage, highlighting the complexity of family relations in later life.

In the last contribution to this section, Hayslip and Page follow a family systems perspective to address the interplay of family members' grief and grieving, extending examination to relationships beyond the immediate family to those in the broader interpersonal and cultural context. They alert us to patterns of similarities and differences in expressions of grief among family members, relating these to the impact of the death, the meaning of the loss and, importantly from the family systems perspective, as a function of (changing) interactions and communication (at different phases of the life cycle) between family members. These dynamic phenomena are considered in relationship to the manifestations and course of individual grief and grieving.

Part 4 covers a range of topics relating to broader consequences and scientific evaluation of family bereavement phenomena. Four illustrations are selected, namely, (1) the financial consequences for the family and the resulting need to extend the scope of research beyond health issues; (2) assessment (i.e. measurement in scientific investigations) of family bereavement phenomena; (3) large-scale assessment of divorce following parental bereavement and (4) the impact within families of the provision of professional intervention and care. First, Corden and Hirst address a less-researched but very important consequence of bereavement, one that can become a major source of stress in the family, that is, the financial ramifications of the death. A number of economic and financial transitions take place, with implications for family members/groups, including lowered income, immediate funeral-related expenses and a legacy of costs deriving from the pre-bereavement caring-giving period. Corden and Hirst review how these features may affect families, drawing on their own qualitative and quantitative UK study, as well as studies conducted in various countries. The authors lead us to consider the role of individual families alongside the responsibilities of formal financial institutions, governments and bereavement services. 
Next, Hooghe, de Mol, Baetens and Zech review the range of assessment tools used to investigate bereavement phenomena at the family level. They cover quantitative and qualitative types of evaluation. They provide a review of both couple and family measurement instruments, describing the content as well as psychometric properties of the available measures. These techniques have gone quite some way toward enabling investigation of ways that an individual's grief influences family functioning and how family dynamics mediate family members' individual grief experiences. The authors give guidelines for extending evaluation techniques for family-level bereavement phenomena and suggest how further qualitative research can be used to provide guidelines for developing more sophisticated family-assessment instruments.

Is the impact of the death of a child in the family of such great impact that the bereaved parents' marriage actually breaks down? There has long been debate about this, with some claiming higher divorce rates, while others state that there is no such impact. One reason for these contradictory conclusions is a severe limitation in the availability of sound empirical evidence. Lyngstad fills an important gap in his contribution to this Special Issue, providing the largest study to date of the impact of death of a child on parents' rates of divorce. He uses longitudinal data from the entire Norwegian population, controlling for a number of important potentially confounding factors, to examine not only the risk of divorce per se, but how this relates to the presence of other children and the duration of bereavement.

Professional intervention programs for bereavement at the family level are few and far between. Clearly, given the detrimental effects for (some) family members, the provision of therapeutic counselling and care from a systems perspective is of particular relevance in our context: How should such programs be designed, what should they cover, for whom are they effective? In the next article, Sandler, Wolchik, Ayers, Tein and Luecken describe their exemplary Family Bereavement Program, which was based on a contextual resilience model. This program was designed for parentally bereaved children and their surviving parent/caregiver. In addition to describing their intervention, the authors summarize a 6-year evaluation of the program using a randomized experimental design, which supports the effectiveness of the program.

In Part 5, Atle and Kari Dyregrov reflect on and appraise this Special Issue, providing their own take on family issues in bereavement research. They put each of the contributions to the Special Issue individually into perspective and give their own general vision on the need for a more social, family approach in bereavement research. Their take on the issues raised reflects their own research and clinical approaches, drawing on years of experience in the bereavement field. They acknowledge the difficulties involved in conducting family-level bereavement research, but stress the need for a sound knowledge base, particularly to provide well-guided (professional) support for bereaved families. Finally, they highlight a major theme that has been raised in different contributions to this volume, namely, the need to embed this type of research within the larger societal context, taking cultural variations into account.

In concluding this outline of the scope and content of our volume, we would like to express the hope that this set of articles provides a further stimulus for discussion and that it furthers the research effort in the family domain. It will have become evident that all authors in this Special Issue are firmly convinced of the imperativeness of extending bereavement research at the family level: bereavement is indeed a family affair! Nevertheless, we would like to emphasize our belief that there is a parallel interest: it is equally important to try to understand what goes on within individuals when they are dealing with dying and death and when they are coming to terms with their bereavement. As such, there remains an important place for the study of individual grief and grieving in scientific enquiry. But our aim in compiling the articles in this Special Issue is to bring home the vital importance of family phenomena as well; in our view, they have demonstrated many powerful ways that living and grieving within family contexts impact on adjustment to the loss of a loved one. It is, then, our belief that scientific understanding needs to span both individual - intrapersonal - and family - interpersonal domains. Hopefully, there will ultimately be mutual understanding, even synthesis, between the two, leading to a whole being greater than the sum of its parts. 